\title{
Large Magnetoresistance and Jahn-Teller Effect in $\mathrm{Sr}_{2} \mathrm{FeCoO}_{6}$
}

Pradheesh R. ${ }^{1 *}$, , Harikrishnan S. Nair ${ }^{2}$, V. Sankaranarayanan ${ }^{1 *}$, and K. Sethupathi ${ }^{1}{ }^{*}$

${ }^{1}$ Low Temperature Physics Laboratory, Department of Physics, Indian Institute of Technology Madras, Chennai 600036, India.

2 Jülich Center for Neutron Sciences 2/ Peter Grünberg Institute 4, Forschungszentrum Jülich GmbH, 52425 Jülich, Germany.

May 29, 2012

\begin{abstract}
Neutron diffraction measurement on the spin glass double perovskite $\mathrm{Sr}_{2} \mathrm{FeCoO}_{6}$ reveals site disorder as well as $\mathrm{Co}^{3+}$ intermediate spin state. In addition, multiple valence states of $\mathrm{Fe}$ and Co are confirmed through Mössbauer and X-ray photoelectron spectroscopy. The structural disorder and multiple valence lead to competing ferromagnetic and antiferromagnetic interactions and subsequently to a spin glass state, which is reflected in the form of an additional $T$-linear contribution at low temperatures in specific heat. A clear evidence of Jahn-Teller distortion at the $\mathrm{Co}^{3+}{ }_{-} \mathrm{O}_{6}$ complex is observed and incorporating the physics of Jahn-Teller effect, the presence of localized magnetic moment is shown. A large, negative and anomalous magnetoresistance of $\approx 63 \%$ at $14 \mathrm{~K}$ in $12 \mathrm{~T}$ applied field is observed for $\mathrm{Sr}_{2} \mathrm{FeCoO}_{6}$. The observed magnetoresistance could be explained by applying a semi-empirical fit consisting of a negative and a positive contribution and show that the negative magnetoresistance is due to spin scattering of carriers by localized magnetic moments in the spin glass phase.
\end{abstract}

PACS. 7 1.70.Ej, 73.43.Qt, 71.23.An, 75.50.Lk

\section{Introduction}

The rich physical properties of $A_{2} M M^{\prime} \mathrm{O}_{6}(A=$ alkaline earth; $M, M^{\prime}=$ transition metal) double perovskites are well documented in the review article by Serrate et. al., [1. One of the most-studied system in this respect is the ferromagnet $\mathrm{Sr}_{2} \mathrm{FeMoO}_{6}$ (SFMO) [2, which exhibits mag- netoresistance at elevated temperature of about $420 \mathrm{~K}$. Structurally ordered as well as disordered SFMO were studied by Sarma et al., 2] who observed the existence of sharp changes in low-field MR in the case of ordered sample. Cobalt-doped $\mathrm{Sr}_{2} \mathrm{FeMoO}_{6}$ also exhibits an appreciable MR (40\%) which was attributed to the inhomo- 
geneous magnetic state [3. Structural defects like antisite disorder, antiphase boundaries and oxygen stoichiometry lead to competing magnetic phases in double perovskites 2,4]. For example, magnetic spin glass phase stemming from intrinsic disorder was reported in nanoscale powders of $\mathrm{Sr}_{2} \mathrm{FeMoO}_{6}[$ ].

Most of the experimental investigations on double perovskite oxides where focused on compounds in which $M$ and $M^{\prime}$ were a combination of first and third row transition metals (TM). Recently, the studies aimed at combining the first-row TMs (Co and Mn, for example) have pointed towards the importance of valence states of the TMs in these systems [6]. For example, $\mathrm{Mn}^{3+}$ and $\mathrm{Co}^{3+}$ can have intermediate spin states (IS) which can lead to Jahn-Teller (JT) distortions where the JT ion will have a single $e_{g}$ electron and the double degeneracy of $e_{g}$ state will be lifted by the distortion [7. This will lead to itinerant behaviour of the single electron which will contribute to magnetic and transport properties.

There are only a few reports on experimental investigations on the Co based double perovskite, $\mathrm{Sr}_{2} \mathrm{FeCoO}_{6} 8$ 9. Ab initio band structure calculations on $\mathrm{Sr}_{2} \mathrm{FeCoO}_{6}$ showed that both Co and Fe make comparable contributions to ferromagnetism of the compound [10 where $\mathrm{Co}^{4+}$ and $\mathrm{Fe}^{4+}$ in high spin states can lead to metallicity and ferromagnetism. However, owing to the comparable ionic radii and valence states of $\mathrm{Fe}$ and $\mathrm{Co}$ (both in $4+$ state), it is surprising that this material showed ferromagnetism. Recently, Co-based double perovskites have attracted interest also due to the excellent electrochemi- cal performance as anode materials in solid oxide fuel cells [11. In our previous investigation on the magnetic properties of $\mathrm{Sr}_{2} \mathrm{FeCoO}_{6}$, [12] clear signatures of spin glass state originating from structural disorder and multiple valence states of $\mathrm{Fe} / \mathrm{Co}$ were obtained which motivated us to investigate its possible consequences on the magnetoresistive properties. In the present article we report a comprehensive study on the structure, Mössbauer and X-ray photoelectron spectroscopy, specific heat and magnetoresistance of $\mathrm{Sr}_{2} \mathrm{FeCoO}_{6}$.

\section{Experimental}

$\mathrm{Sr}_{2} \mathrm{FeCoO}_{6}$ samples used in this study were prepared by sol-gel method as described elsewhere [12,13]. Neutron powder diffraction (NPD) measurements at 12, 30, 60, 65 and $300 \mathrm{~K}$ were performed with neutron wavelength of $1.4789 \AA$ employing position sensitive detector. The crystal structure was refined by Rietveld method [14] using FULLPROF program [15]. Mössbauer measurements in transmission mode were performed with ${ }^{57} \mathrm{Co}$ source in constant acceleration mode using a Mössbauer spectrometer equipped with a Weissel velocity drive. The spectrum was collected at room temperature using Canberra S-100 constant-acceleration Mössbauer spectrometer having the ${ }^{57} \mathrm{Co}$ in Rh matrix. The velocity was calibrated using the spectra of $\alpha$-Fe foil. The software Fit; O was used to analyze the Mössbauer spectrum [16]. Electron core level binding energy was analyzed using Philips X-ray photoelectron spectrometer (PHI5700). Specific heat measurements were performed using a semi-adiabatic heat pulse 


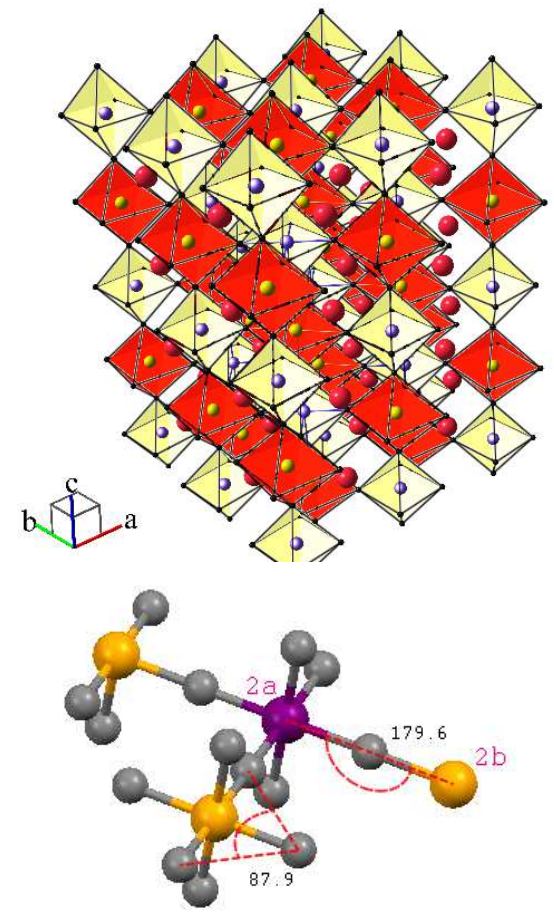

Fig. 1. (colour online) Top: A schematic of the crystal structure of $\mathrm{Sr}_{2} \mathrm{FeCoO}_{6}$. Bottom: $\mathrm{FeO}_{6}$ and $\mathrm{CoO}_{6}$ octahedra are shown, where Fe are orange and Co purple. The tilt $\left(\phi_{1}=179.64\right)$ and rotation $\left(\phi_{2}=87.94\right)$ angles at $12 \mathrm{~K}$ are also shown.

technique in the temperature range of $4-300 \mathrm{~K}$ at $0 \mathrm{~T}$ and applied field of $8 \mathrm{~T}$. Electrical resistivity in $0 \mathrm{~T}$ and applied magnetic field of $6 \mathrm{~T}$ was measured employing standard four-probe method on circular pellets of $\mathrm{Sr}_{2} \mathrm{FeCoO}_{6}$. Magnetoresistance measurements were carried out using a $12 \mathrm{~T}$ superconducting magnet in an exchange cryostat.

\section{Results and Discussion}

\subsection{Neutron diffraction}

The analysis of neutron diffraction data was performed using tetragonal $I 4 / m$ space group, presented in figure 2 A completely random occupancy of $\mathrm{Fe}$ and $\mathrm{Co}$ in the
Table 1. Structural parameters of $\mathrm{Sr}_{2} \mathrm{FeCoO}_{6}$ at different temperatures. The Wyckoff positions are $\mathrm{Sr} 4 d\left(0, \frac{1}{2}, \frac{1}{4}\right) ; \mathrm{Fe} 1$ $2 a(0,0,0) ; \mathrm{Fe} 22 b\left(0,0, \frac{1}{2}\right) ; \mathrm{Co} 12 b\left(0,0, \frac{1}{2}\right) ; \mathrm{Co} 22 a(0,0,0) ; \mathrm{O}_{a p}$ $4 e(0,0, z)$ and $\mathrm{O}_{e q} 8 h(x, y, z)$. The agreement factors of the refinement are quantified as $\chi^{2}$ and $R_{\text {Bragg }}$.

\begin{tabular}{|c|c|c|c|c|c|}
\hline & & $12 \mathrm{~K}$ & $30 \mathrm{~K}$ & $65 \mathrm{~K}$ & $300 \mathrm{~K}$ \\
\hline Space Group & & $I 4 / \mathrm{m}$ & $I 4 / m$ & $I 4 / m$ & $I 4 / m$ \\
\hline$a(\AA)$ & & $5.4360(1)$ & $5.4390(2)$ & $5.4381(1)$ & $5.4554(3)$ \\
\hline$c(\AA)$ & & $7.7010(3)$ & $7.6982(3)$ & $7.7037(3)$ & $7.7319(1)$ \\
\hline$V\left(\AA^{3}\right)$ & & $227.569(3)$ & $227.736(2)$ & $227.826(1)$ & $230.115(5)$ \\
\hline $\mathrm{O}_{a p}$ & $4 e(0,0, \mathrm{z})$ & & & & \\
\hline$z$ & & $0.2592(1)$ & $0.2502(2)$ & $0.2495(1)$ & $0.2530(4)$ \\
\hline $\mathrm{O}_{e q}$ & $8 h(\mathrm{x}, \mathrm{y}, 0)$ & & & & \\
\hline$x$ & & $0.2491(9)$ & $0.2548(1)$ & $0.2581(9)$ & $0.2498(2)$ \\
\hline$y$ & & $0.2507(6)$ & $0.2433(7)$ & $0.2400(3)$ & $0.2541(7)$ \\
\hline$\chi^{2}$ & & 3.5 & 3.4 & 3.37 & 3.83 \\
\hline$R_{\text {Bragg }}$ & & 4.2 & 4.2 & 3.97 & 3.63 \\
\hline
\end{tabular}

$2 a(0,0,0)$ and $2 b\left(0,0, \frac{1}{2}\right)$ sites, as previously done in the case of X-ray investigations [12, were assumed in the refinement procedure. The refined lattice parameters, unit cell volume and atomic positions of $\mathrm{Sr}_{2} \mathrm{FeCoO}_{6}$ (SFCO) determined from the Rietveld analysis are presented in Table 1. A completely disordered arrangement of cations were also reported in the case of double perovskites like $\mathrm{Sr}_{2} \mathrm{MnNbO}_{6}, \mathrm{Sr}_{2} \mathrm{MnRuO}_{6}$ [17, where $I 4 / m c m$ space group also was suggested for a completely disordered cationic arrangement. Hence, refinement of the structure in $14 / \mathrm{mcm}$ space group with $\operatorname{Sr}(4 b)\left(0, \frac{1}{2}, \frac{1}{4}\right) ; \mathrm{Fe} / \mathrm{Co}(4 c)(0,0,0) ; \mathrm{O}(4 a)$ $\left(0,0, \frac{1}{4}\right) ; \mathrm{O}(8 h)\left(x, x+\frac{1}{2}, 0\right)$ was also performed for SFCO. When the octahedral volume is occupied by 50:50 mixture of cations, the most probable tilt system is $a^{0} a^{0} a^{-}$ 


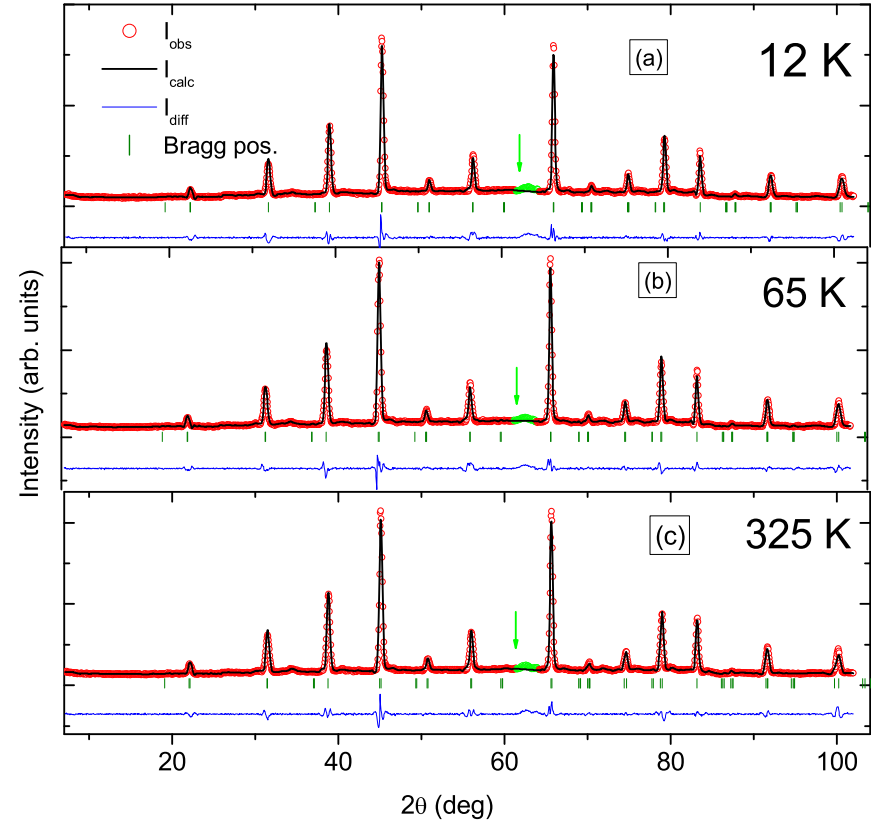

Fig. 2. (colour online) Neutron powder diffraction patterns of $\mathrm{Sr}_{2} \mathrm{FeCoO}_{6}$ at (a) $12 \mathrm{~K}$, (b) $65 \mathrm{~K}$ and (c) $325 \mathrm{~K}$ are presented along with the results of Rietveld refinement. The structure was refined in $I 4 / m$ space group at all temperatures. In the figure, a vertical down-arrow (coloured green) indicates the region of $2 \theta=61.4$ to $64.1^{\circ}$ which was excluded from the refinement.

and the space group being $I 4 / m$ if the cations are ordered or $I 4 / \mathrm{mcm}$ if they are disordered. However, in our analysis reproduction of the observed intensities and better agreement factors were accomplished with the choice of $I 4 / m$.

Previous macroscopic magnetic measurements on $\mathrm{Sr}_{2} \mathrm{FeCoO}_{6}$ [12 revealed that the valence state of Co was +3 and the spin state as IS (intermediate spin). IS $\mathrm{Co}^{3+}$ is a JahnTeller active ion and hence it distorts the $\mathrm{Co}-\mathrm{O}_{6}$ octahedra to further remove the degeneracy of the triply degenerate $\mathrm{t}_{2 g}$ and doubly degenerate $\mathrm{e}_{g}$ states. The experimentally determined bond lengths for SFCO, given in Table2, show the presence of two long and four short bond lengths for $\mathrm{CoO}_{6}$ and two short and four long bonds for $\mathrm{FeO}_{6}$ suggesting the distortion of $\mathrm{CoO}_{6}$ octahedra owing to Jahn-Teller (JT) activity. The bond distances Fe-O increase with in-

Table 2. Main bond distances $(\AA)$ and selected angles (deg) of $\mathrm{Sr}_{2} \mathrm{FeCoO}_{6}$ at different temperatures. The distortion parameter of the octahedra is defined as $\Delta_{d}=(1 / 6) \Sigma_{n=1-6}\left[\left(d_{n}-<\right.\right.$ $d>) /\langle d\rangle]^{2} .\langle d\rangle$ is the average $\mathrm{Mn}-\mathrm{O}$ distance. The strain parameter is $e_{t}=\left(c_{p}-a_{p}\right) /\left(c_{p}+a_{p}\right) . c_{p}$ and $a_{p}$ are the equivalent primitive perovskite lattice constants given by $c / 2$ and $a / \sqrt{(2)}$ respectively. The structural parameters in the absence of distortions as calculated using SPuDS are also shown.

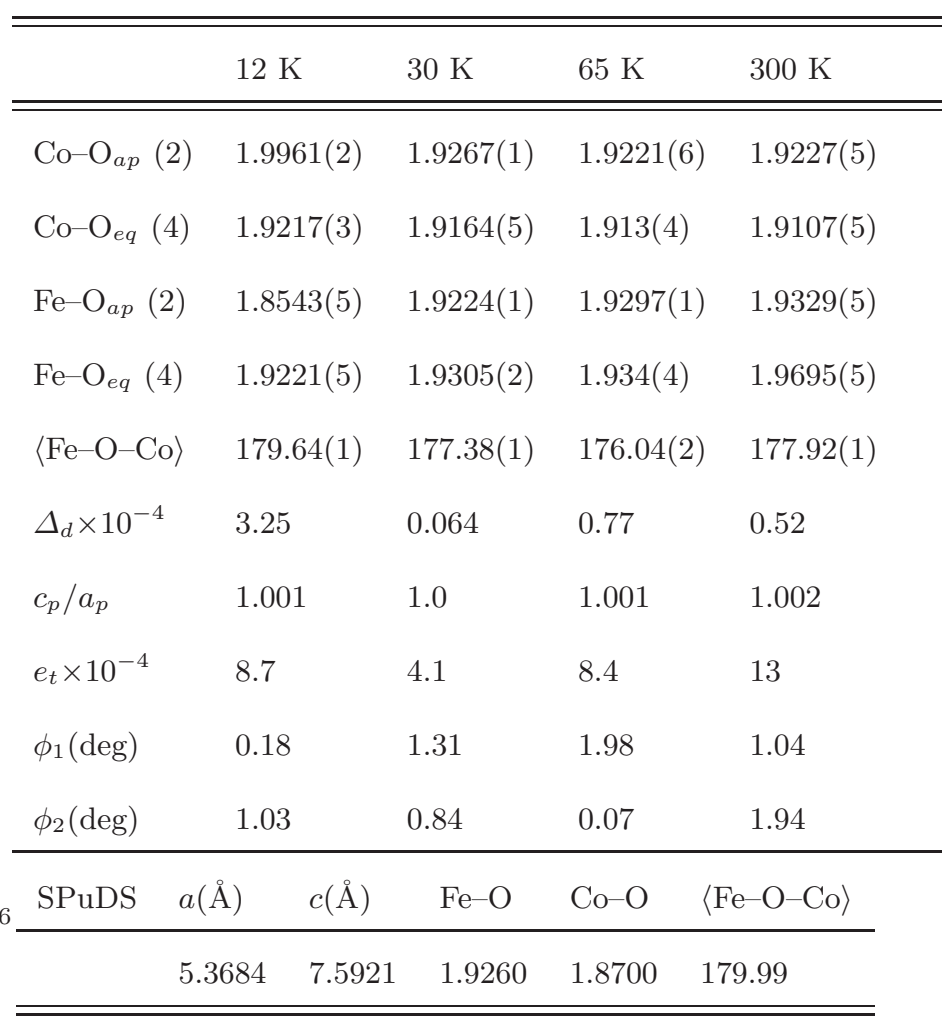

creasing temperature while $\mathrm{Co}-\mathrm{O}$ distances show the opposite trend. The Jahn-Teller distortion has three sets of modes namely, breathing mode $\left(Q_{1}\right)$, basal distortion $\left(Q_{2}\right)$ and octahedral stretching $\left(Q_{3}\right)$. From the bond length cal- 
culation, two long and four short, it is obvious that $\mathrm{Co}^{3+}$ shows $Q_{3}$ distortion mode 18 . The distortions of the octahedra are reflected in the octahedral tilting of $M \mathrm{O}_{6}$ from the $z$ axis defined as $\phi_{1}=(180-\Theta) / 2$ where $\Theta$ is the $M-\mathrm{O}-M^{\prime}$ bond angle along $z$ axis. The rotation angle in the $x y$-plane, defined as $\phi_{2}=(90-\Omega) / 2$ where $\Omega$ is the angle among three oxygen ions between two corner-shared oxygen octahedra, also quantifies the degree of distortion. The tilt angle $\phi_{1}$ and the rotation angle $\phi_{2}$ of SFCO at different temperatures are given in Table 2, The values of average bond angles $<\mathrm{Fe}-\mathrm{O}-\mathrm{Co}>$ and the distances of $\mathrm{Fe}-\mathrm{O}$ and $\mathrm{Co}-\mathrm{O}$ bonds and various distortion parameters are also presented in the table. They are not as large compared to the values observed in double perovskites like $\mathrm{Sr}_{2} \mathrm{MnSbO}_{6}[19]$ however, $\phi_{1}\left(\phi_{2}\right)$ shows high (low) values in the intermediate temperature range where an anomaly in lattice constants were reported earlier [12]. The octahedral tilting and the distortions are also quantified through empirical parameters like, $c_{p} / a_{p}\left(c_{p}=c / 2\right.$ and $\left.a_{p}=a / \sqrt{2}\right)$ which denote the ratio of equivalent primitive perovskite lattice parameters; and the distortion parameter $\Delta_{d}$, defined as $(1 / 6) \Sigma_{n=1-6}\left[\left(d_{n}-<d>\right) /<d>\right]^{2}$. Typical values of these parameters for SFCO are given in Table 2 From Table 2, it is clear that the tetragonal strain defined as, $e_{t}=\left(c_{p}-a_{p}\right) /\left(c_{p}+a_{p}\right)$, also shows an increase with increasing temperature. In order to signify the distortions of the octahedra, we have calculated the lattice parameters and the bond parameters at room temperature using the program SPuDS 20] (see, Table 2). A comparison between the ideal values calculated by SPuDS and the experimen- tally determined values for SFCO indicates the major role played by lattice distortions in this double perovskite.

\subsection{Mössbauer and X-ray Photoelectron Spectroscopy}
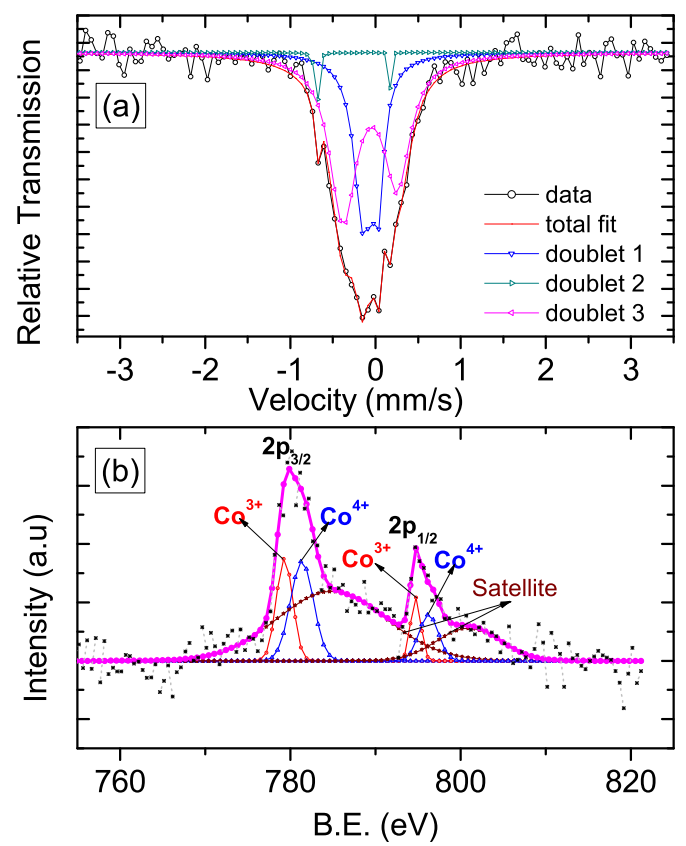

Fig. 3. (color online) (a) Room temperature Mössbauer spectra of $\mathrm{Sr}_{2} \mathrm{FeCoO}_{6}$. The experimental data are shown in black circles and the total fit assuming three doublets is presented as red solid line. Separate contributions from the three doublets are also shown. (b) The Co-XPS spectra of $\mathrm{Sr}_{2} \mathrm{FeCoO}_{6}$ and the fits for $\mathrm{Co}^{3+}$ and $\mathrm{Co}^{4+}$. The black dashed line and squares represents experimental data points and the solid magenta line represents the fitted curve.

Cationic disorder and mixed valence of the transition metals $\mathrm{Fe}$ and Co results in inhomogeneous magnetic exchange interactions leading to magnetic frustration and spin glass state in SFCO. In our earlier work, using structural and bulk magnetic investigations, has been confirmed 
Table 3. The Mössbauer parameters, isomer shift $(\delta)$ quadrupolar shift $(\Delta)$ and the area ratio of $\mathrm{Sr}_{2} \mathrm{FeCoO}_{6}$ at room temperature.

\begin{tabular}{cccc}
\hline Doublet & $\delta\left(\mathrm{mm} \mathrm{s}^{-1}\right)$ & $\Delta\left(\mathrm{mm} \mathrm{s}^{-1}\right)$ & Area Ratio \\
\hline \hline 1 & $0.1788(3)$ & $0.2009(2)$ & $50.58 \%$ \\
2 & $0.1118(2)$ & $0.6322(2)$ & $28.26 \%$ \\
3 & $0.2809(3)$ & $0.5524(1)$ & $21.16 \%$ \\
\hline \hline
\end{tabular}

the presence of spin glass state in this material [12. However, a clear picture regarding the valence and spin states of cations has not emerged. A clear understanding regarding the valency of the cations and ligand coordination can be obtained from Mössbauer experiments since it probes the local environment of the cations and has been successfully used as a tool to explore the magnetism and electronic structure of the colossal magnetoresistive perovskites 21. The experimentally obtained room temperature Mössbauer spectrum of SFCO is presented in Fig. 3(a). The spectra shows paramagnetic behaviour and is deconvoluted in to three doublets with different isomer shifts and quadrupole splitting. On the basis of theoretical calculations using the difference in the electron density at the absorber and source, it was determined that the $\mathrm{Fe}^{4+}$ in low-spin (LS) state will have an isomer shift $(\delta)$ value in the range $0.1-0.2 \mathrm{~mm} / \mathrm{s}$ [22. The isomer shift, $\delta$, and quadrupole splitting of the paramagnetic components, $\Delta$, determined using the least square fit are given in Table 3 . from which we found that the isomer shift for doublet 2 is in good agreement with that of low-spin $\mathrm{Fe}^{4+}$ and doublet 3 for $\mathrm{Fe}^{3+}$ in an octahedral coordination [23]. In compari- son, the $\delta$ value of doublet 1 is intermediate to that of $\mathrm{Fe}^{4+}$ and $\mathrm{Fe}^{3+}(>0.30 \mathrm{~mm} / \mathrm{s})$ and hence is attributed to $\mathrm{Fe}^{3+y}$ due to the rapid ion exchange as commonly observed in ferrite structures at ordinary temperatures 24. Thus, possibility of a charge disproportionation of the form $2 \mathrm{Fe}^{4+} \rightarrow$ $\mathrm{Fe}^{(4-d)+}+\mathrm{Fe}^{(4+d)+}$ cannot be ruled out 25 . For a comparison, Mössbauer spectra of $\mathrm{Sr}_{2} \mathrm{FeTeO}_{6}$ [26] was fitted to two symmetric doublets with equal isomer shift values of $0.435(2)$ and $0.434(1) \mathrm{mm} / \mathrm{s}$ and quadrupolar splitting of 0.23 and $1.21 \mathrm{~mm} \mathrm{~s}^{-1}$ which are consistent with high spin $\mathrm{Fe}^{3+}$. The scenario of multiple valence states for $\mathrm{Fe}$ and Co in SFCO is also supported by X-ray photoelectron spectroscopy (XPS). Figure 3(b) presents the experimentally observed XPS spectrum of Co in SFCO deconvoluted into Gaussian components which results in an apparent doublet for Co $2 p_{1 / 2}$ and Co $2 p_{3 / 2}$. From the fit, the doublet positions are obtained at 779.64(3) and 781.36(3) eV for Co $2 p_{1 / 2}$ and 794.86(4) and 796.24(3) eV for Co $2 p_{3 / 2}$ with shake-up peaks at $788.63 \mathrm{eV}$ and $800.70 \mathrm{eV}$. The binding energies of $779.64(3) \mathrm{eV}$ and $794.86(4) \mathrm{eV}$ are attributed to $\mathrm{Co}^{3+}$ while $781.36(3) \mathrm{eV}$ and $796.24(3) \mathrm{eV}$ to $\mathrm{Co}^{4+}$ since $\mathrm{Co}^{4+}$ has greater electron density than $\mathrm{Co}^{3+}$ [27. The presence of shake-up satellite for $\mathrm{Co}^{3+}$ confirms that it is not in a low spin state since LS has $t_{2 g}$ states completely filled minimizing the possibility of metal to ligand charge transfer and shake-up peaks. Table 4 shows a comparison of the valence states estimated for SFCO with those of other perovskite cobaltites. Based on the results from Mössbauer and XPS experiments and combining our previous results from magnetic measurements, we can ar- 


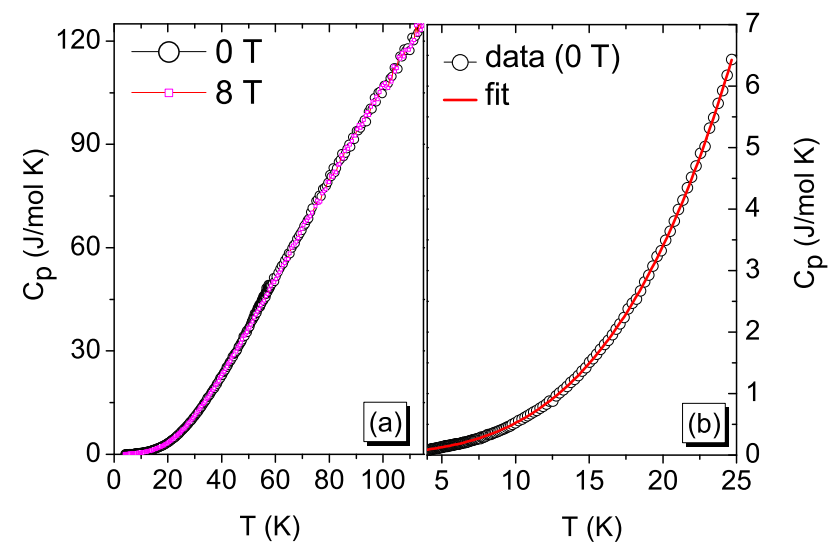

Fig. 5. (a) The specific heat $C_{p}(T)$ of $\mathrm{Sr}_{2} \mathrm{FeCoO}_{6}$ in zero magnetic field and an applied field of 8 T. (b) The low temperature part of the specific heat at $0 \mathrm{~T}$ along with the fit using eqn. 1

Table 4. Binding energy (BE) of Co $2 p$ peaks in $\mathrm{Sr}_{2} \mathrm{FeCoO}_{6}$ compared with other cobaltites. Here, Oct. stands for octahedral environment while LBE and HBE are lower binding energy $\left(2 p_{3 / 2}\right)$ and higher binding energy $\left(2 p_{1 / 2}\right)$. Co is in octahedral coordination in all compounds.

\begin{tabular}{ccccc}
\hline & & \multicolumn{2}{c}{ B.E. } & \\
\cline { 3 - 4 } Oxide & Ion & $2 p_{3 / 2}$ & $2 p_{1 / 2}$ & Reference \\
\hline $\mathrm{Sr}_{2} \mathrm{FeCoO}_{6}$ & $3+$ & $779.61(3)$ & $794.86(4)$ & present work \\
$\mathrm{LiCoO}_{2}$ & $3+$ & 779.5 & 794.6 & [28] \\
$\mathrm{Co}_{3} \mathrm{O}_{4}$ & $3+$ & 779.6 & 794.5 & 20] \\
$\mathrm{La}_{1-x} \mathrm{Sr}_{x} \mathrm{CoO}_{3-\delta}$ & $3+$ & 779.6 & 794.9 & 30] \\
$\mathrm{LaCoO}_{3}$ & $3+$ & 780 & 794.5 & 31] \\
$\mathrm{Sr}_{2} \mathrm{FeCoO}_{6}$ & $4+$ & $781.36(3)$ & $796.24(3)$ & present work \\
$\mathrm{Ba}_{0.5} \mathrm{Sr}_{0.5} \mathrm{Co}_{0.8} \mathrm{Fe}_{0.2} \mathrm{O}_{3-\delta}$ & $4+$ & 780.29 & 795.63 & 27] \\
\hline \hline
\end{tabular}

rive at the possible combinations of different valence states for Fe and Co in SFCO. In Fig. 4 (a), a schematic of the possible spin states that explains the properties of SFCO is presented.

\subsection{Specific Heat}

The temperature dependent specific heat, $C_{p}(T)$, in the range $4-120 \mathrm{~K}$ at an applied field of $0 \mathrm{~T}$ as well as $8 \mathrm{~T}$

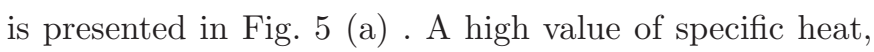

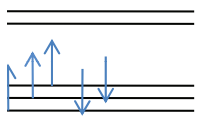

$\mathrm{Fe}^{3+}$

LS

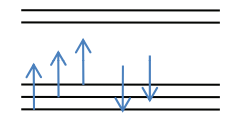

$\mathrm{CO}^{4+}$

LS

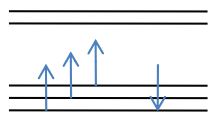

$\mathrm{Fe}^{4+}$

LS

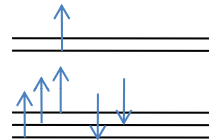

$\mathrm{CO}^{3+}$

IS
Fig. 4. (color online) Schematic of the level schemes of various spin state for different valences of $\mathrm{Fe}$ and $\mathrm{Co}$ in $\mathrm{Sr}_{2} \mathrm{FeCoO}_{6}$. The spin states are indicated as $I S=$ intermediate spin, $L S=$ low spin.

comparable in magnitude to that observed in insulating manganites $\mathrm{Dy}_{0.5} \mathrm{Sr}_{0.5} \mathrm{MnO}_{3}$ [32, $\mathrm{La}_{0.8} \mathrm{Ca}_{0.2} \mathrm{MnO}_{3}$,33], is observed in SFCO also. No anomaly corresponding to the spin glass transition at $T_{c} \sim 75 \mathrm{~K}[12]$ is observed in $C_{p}(T)$ which clearly marks the absence of any collective thermodynamic transition or long range order. It is clear from the figure that even with the application of $8 \mathrm{~T}$ there is no appreciable change in the specific heat. We have tried to model the experimentally observed specific heat, and a satisfactory fit to the experimental data was obtained using a model that includes a linear term in $T$ which accounts for the spin glass contribution, as represented by eqn. 1. The fit achieved in the temperature range 4-25 K is shown in Fig. 5 (b).

$$
C(T)=\gamma T+\beta_{3} T^{3}+\beta_{5} T^{5}
$$

Spin glass systems show a strong linear dependence of specific heat at low temperatures 34,35$]$ and moreover the linear dependence was verified using a full mean field solution of a quantum Heisenberg spin glass model in large $N$ 
Table 5. The fit-parameters obtained from the specific heat analysis performed using eqn. 1]

\begin{tabular}{|l||l|}
\hline \hline$\gamma\left(\mathrm{J} \mathrm{mol}^{-1} \mathrm{~K}^{-2}\right)$ & $0.01655(2)$ \\
$\beta_{3}\left(\mathrm{~J} \mathrm{~mol}^{-1} \mathrm{~K}^{-4}\right)$ & $3.4916(1) \times 10^{-4}$ \\
$\beta_{5}\left(\mathrm{~J} \mathrm{~mol}^{-1} \mathrm{~K}^{-6}\right)$ & $8.5383(1) \times 10^{-8}$ \\
$\Theta_{D}(\mathrm{~K})$ & 409 \\
\hline \hline
\end{tabular}

limit [36]. The disordered nature and the magnetic glassy state, as in the case of manganites [37, also contribute to linear $T$ dependence of specific heat. Resistivity measurements, discussed below (Fig. 6] (a)), show that SFCO is an insulator with the low temperature resistance reaching a value of the order of $10^{6} \Omega \mathrm{cm}$ and hence the $T$-linear term that we observe in $C_{p}(T)$ originates from the spin degrees of freedom and disorder. The coefficient of linear term, $\gamma$, obtained from the analysis was $16 \mathrm{~mJ} \mathrm{~mol}^{-1}$ $\mathrm{K}^{-2}$ which is close to $11 \mathrm{~mJ} \mathrm{~mol}^{-1} \mathrm{~K}^{-2}$ observed in the case of La-doped $\mathrm{Sr}_{2} \mathrm{FeMoO}_{6}$ [38. The Debye temperature $\Theta_{D}=409 \mathrm{~K}$ was estimated for SFCO using the relation $\Theta_{D}=\left(12 \pi^{4} p R / 5 \beta_{3}\right)^{1 / 3}$, where $R$ is the gas constant and $p$ is the number of atoms. The specific heat coefficient $\beta_{3}$ is extracted from the fit. The Debye temperature estimated is comparable to the value $\Theta_{D}=463 \mathrm{~K}$ [12] from Grüneisen approximation for the unit cell volume. The specific heat investigations on $\mathrm{Sr}_{2} \mathrm{FeMoO}_{6}$, for example, have reported a $\Theta_{D}$ value of $338 \mathrm{~K}[39$, which lies close to the value obtained for SFCO. A detailed analysis of the specific heat data of $\mathrm{Sr}_{2} \mathrm{FeTeO}_{6}$ using two different Debye temperatures for the light and heavy atoms resulted in the values of $768 \mathrm{~K}$ and $261 \mathrm{~K}$ respectively [26]. Attempts to analyze the $C_{p}(T)$ data including a $T^{3 / 2}$ term in or- der to take in to account the antiferromagnetic spin wave [40] were also made. However, the fitting was unfavourable due to the presence of powers of $T$ with comparable magnitudes. The value of $\gamma$ obtained from the fit is due to the spin glass nature and is comparable to the values observed in spin glass 41] and cluster glass perovskites 42.

\subsection{Magnetoresistance}

The electrical resistance of SFCO in 0 and $6 \mathrm{~T}$ applied magnetic field is presented in Fig. 6 (a). It exhibits a monotonic increase as the temperature is lowered below the spin glass transition temperature. The variation of electrical resistance indicates semiconducting behaviour. The inset of the figure shows the log-log representation of the main panel and suggests that thermally activated behaviour of carrier hopping does not hold in SFCO. The resistivity does not follow Arrhenius law and a real energy gap opening being the reason for the upturn of resistance at low temperature is ruled out since no divergence in the derivative of resistivity is observed. Colossal magnetoresistance effects are normally observed at the transition temperatures (metal-insulator or magnetic) but in the case of SFCO, maximum MR is found at very low temperatures. The temperature evolution of $\mathrm{MR}$ is presented in Fig. 6 (b). The MR is positive for a small range at high temperatures and then begins to decrease with decreasing temperature and goes to negative values. Negative magnetoresistance observed in semiconductors was compared with the effects observed in dilute alloys such as $\mathrm{Cu}-\mathrm{Mn}$ 

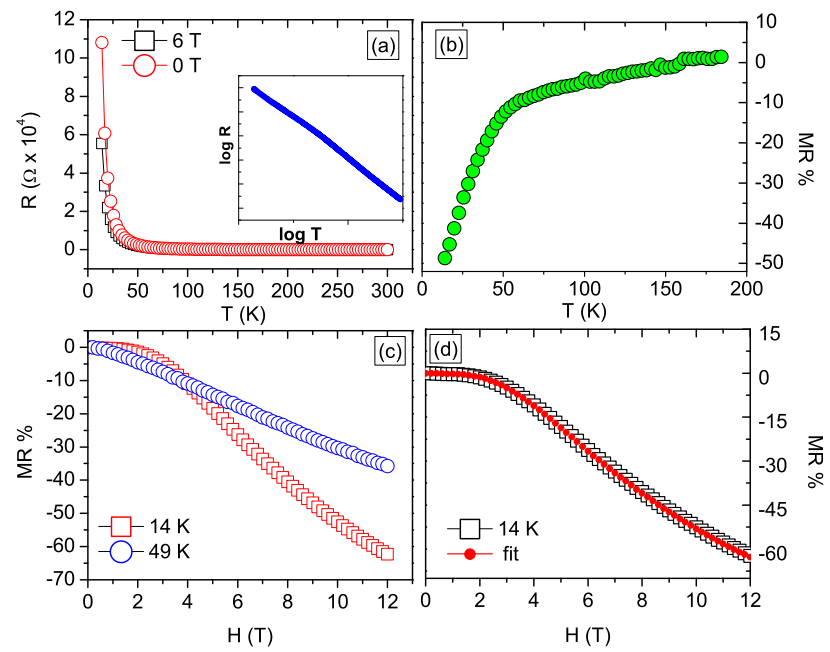

Fig. 6. (colour online) (a) Temperature dependence of electrical resistance of $\mathrm{Sr}_{2} \mathrm{FeCoO}_{6}$ in zero field and an applied field of $6 \mathrm{~T}$. Inset shows the zero field resistance in log-log scale. (b) Magnetoresistance of $\mathrm{Sr}_{2} \mathrm{FeCoO}_{6}$ as a function of temperature. (c) Magnetoresistance as a function of applied magnetic field at two temperatures, $14 \mathrm{~K}$ and $49 \mathrm{~K}$. (d) Fit to the isothermal MR at $14 \mathrm{~K}$ using eqn. (2). The fit parameters obtained are, $A_{1}=0.1086(4), A_{2}=0.0013(4), B_{1}=0.0159(3)$ and $n=1.43(6)$.

and a theory based on localized magnetic moments was proposed by Toyazawa 43. Later, in an empirical model suggested by Khosla and Fischer [44], a negative component to magnetoresistance stemming from the localized magnetic moment model of Toyazawa and a positive component due to the field dependence of Hall coefficient were combined to explain the data on CdS. In order to explain the observed magnetoresistance in SFCO, we employ the empirical relation derived by Khosla and Fischer. Thus, the isothermal MR was found to fit to the following expression,

$$
\frac{\Delta \rho}{\rho}=-A_{1}^{2} \ln \left(1+{A_{2}}^{2} H^{2}\right)+\frac{B_{1}^{2} H^{n}}{1+B_{2}^{2} H^{2}}
$$

where $A_{1}, A_{2}, B_{1}, B_{2}$ and $n$ are free parameters of fitting and $\mathrm{H}$ is the applied magnetic field. $A_{1}$ and $A_{2}$ are given by

$$
\begin{array}{r}
A_{1}{ }^{2}=a^{2} J\left[S(S+1)+\left\langle M^{2}\right\rangle\right] \\
A_{2}{ }^{2}=\left[1+4 S^{2} \pi^{2}\left(\frac{2 J \rho_{F}}{g}\right)^{4}\right] \frac{g^{2} \mu^{2}}{\left(\alpha k_{B} T\right)^{2}}
\end{array}
$$

where $a$ is regarded as a measure of spin scattering. The positive component of MR is proportional to $H^{n}$ where $n$ ranges from 0.77 to 2.19 . A value less than 1.8 has been observed for systems showing a monotonic increase in negative MR [45]. The field-dependent MR of SFCO at $14 \mathrm{~K}$ and $49 \mathrm{~K}$ are presented in Fig. 6 (c) and the data at $14 \mathrm{~K}$ along with the fit using equation (2) are presented in Fig.6 (d). The value of $n=1.43(6)$ obtained from this fit lies in the range consistent with negative MR.

Owing to crystal field effects, the five-fold degenerate $3 d$ level of $\mathrm{Co}^{3+}$ splits into three-fold degenerate $t_{2 g}$ and twofold degenerate $e_{g}$ states. The system may prefer further removal of degeneracy through Jahn-Teller distortion, if it can help in lowering the energy. As schematically shown in Fig 4 . only $\mathrm{Co}^{3+}$ has an occupied $e_{g}$ state. It suits the system to undergo Jahn-Teller distortion of the $\mathrm{Co}^{3+}-\mathrm{O}_{6}$ octahedra. Our neutron diffraction measurement confirms the $\mathrm{Co}^{3+}-\mathrm{O}_{6}$ distortion as shown in Fig 2 The distortion is manifested by changing the tilt angle and rotation angle from $180^{\circ}$ and $90^{\circ}$ respectively. However, the presence of a Jahn-Teller ion can lead to a local structural distortion thereby removing the degeneracy of the two-fold $e_{g}$ state. If, through such a degeneracy removal, the upper level of the two-fold $e_{g}$ states is available to an electron, it can strongly influence the conduction mechanism of SFCO. 
From the structural studies it is evident that there is an increase in the bond length along the apical direction which means a descent in the symmetry to $D_{4 h}$. The lowering of the symmetry is treated as a perturbation to the cubic field and in order to link the local structural distortion to the energy level splitting, we note that the energy separation between the $d_{x^{2}-y^{2}}$ and $d_{z^{2}}$ arises from the second order crystal field coefficient of distorted octahedra. Hence the Jahn-Teller coupling $E_{J T}$ can be described as 46

$$
\begin{array}{r}
E_{J T}^{e_{g}}=4 D_{s}+5 D_{t} \\
E_{J T}^{t_{2 g}}=3 D_{s}-4 D_{t} \\
D_{s}=\frac{e}{14} \sqrt{\frac{5}{\pi}} A_{20}\left\langle r^{2}\right\rangle_{3 d} \\
D_{t}=\frac{e}{14 \sqrt{\pi}} T\left\langle r^{4}\right\rangle_{3 d}
\end{array}
$$

where $A_{20}$ is the second order crystal electric field (CEF) coefficient, $T=A_{40}^{\prime}-A_{40}$ is the difference between fourth order CEF coefficients of distorted octahedra and perfect octahedra. $\left\langle r^{n}\right\rangle$ is the expectation value of the $n^{t h}$ power of the radial distance of a $3 d$ orbital from the nucleus. $D_{s}$ and $D_{t}$ are splitting parameters for the spherical harmonics $Y_{2}^{0}$ and $Y_{4}^{0}$ as given in eqn (9) and eqn (10) and $R_{3 d}$ is the normalized spherically symmetric radial functions, $r$ is the radius and $R$ is the $\mathrm{Co}-\mathrm{O}$ bond length.

$$
\begin{aligned}
D_{s} & =\int\left[R_{3 d}(r)\right]^{2} \frac{3}{2} \frac{r^{2}}{R^{3}} \cdot r^{2} d r \\
D_{t} & =\int\left[R_{3 d}(r)\right]^{2} \frac{3}{2} \frac{r^{4}}{R^{5}} \cdot r^{2} d r
\end{aligned}
$$

Using eqn. (9) and eqn. (10) we can arrive at eqn. (7) and eqn. (8). Taking the radius of IS $\mathrm{Co}^{3+}(0.56 \AA)$, the value of $D_{t}$ works out to be less than $D_{s}$. Hence the main contribution to energy arises from $A_{20}$ and $E_{J T}$ can be written as

$$
E_{J T}^{e_{g}} \approx 4 D_{s}=\frac{2 e}{7} \sqrt{\frac{5}{\pi}} A_{20}\left\langle r^{2}\right\rangle_{3 d}
$$

The radius of $\mathrm{Co}^{3+}$ in IS state is nearly equal to that of $\mathrm{Fe}^{3+}(0.55 \AA)$ and hence the second order CEF coefficient can be evaluated using the quadrupole splitting obtained from the Mössbauer data. The quadrupole splitting can be expressed as $\Delta=\frac{e Q V_{z z}}{2}\left(1+\frac{\eta^{2}}{3}\right)^{1 / 2}$ where $Q$ is the electric quadrupole moment of the ${ }^{57} \mathrm{Fe}$ nucleus, $\eta$ is the asymmetry parameter, and $V_{z z}$ is the principal component of the electric field gradient at the nucleus which contains contributions from valence as well as the surrounding electrons.

$$
\begin{array}{r}
V_{z z}=V_{z z}(F e)+V_{z z}(\text { lattice }) \\
V_{z z}(\text { lattice })=\sum \frac{q_{i}\left(3 \cos ^{2} \theta-1\right)}{R_{i}^{3}}=\sqrt{\frac{5}{\pi}} A_{20}
\end{array}
$$

Combining eqns (11) and (13) and converting $Q$ to eV by a factor of $\frac{E \gamma}{c}$, where $c$ is the velocity of light and using $Q=0.28 \times 10^{-24} \mathrm{~cm}^{2}, \eta=0$ for axial symmetry we get $E_{J T}^{e_{g}}=1.3 \mathrm{eV}$ (note that this is the minimum energy and hence a contribution from $D_{t}$ will only increase the energy). This value is high enough such that the electron in the $e_{g}$ level is not itinerant but localized, consistent with the experimentally observed resistivity. The large energy separation $\mathrm{E}_{J T}$, traps the electron leading to localization; consequently, the ground state is insulating. From Fig 4 we can see that all the other valence states have unoccupied $e_{g}$ levels and as the $t_{2 g}$ states are already localized due to crystal field effect the $\mathrm{Co}-\mathrm{O}-\mathrm{Fe}$ interaction is antiferromagnetic. Due to the tetragonal symmetry, the three-fold 


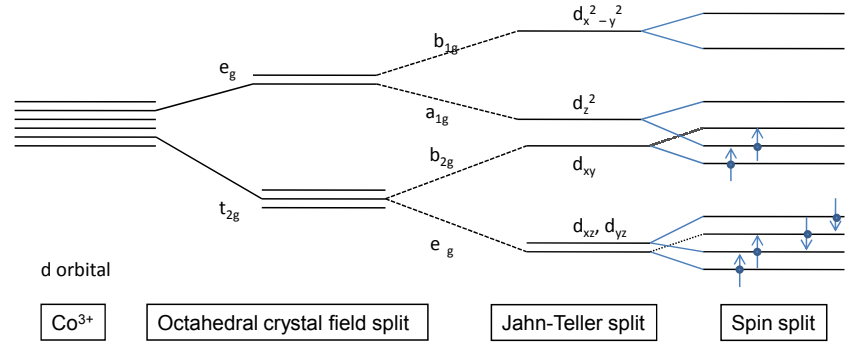

Fig. 7. (colour online) Schematic of $\mathrm{Co}^{3+}$ level scheme in IS state showing the removal of degeneracy.

$t_{2 g}$ state will further split into an $b_{2 g}$ state and a doubly degenerate $e_{g}^{\prime}$ state while the $e_{g}$ state will split into $a_{1 g}$ and $b_{1 g}$ states as shown in Fig. 7. The insulating behaviour of SFCO suggests that the conducting carriers are holes that lie mainly in $a_{1 g}$ state [47. The holes are strongly coupled to lattice and leads to localization resulting in localized magnetic moments due to spin-phonon coupling.

A small contribution of positive $\mathrm{MR}$ is also seen in the present case which is associated with the spin glass behaviour observed in this system. Strong competition occurs between spin clusters and ferromagnetic state below the spin glass transition temperature. As an external magnetic field is applied the ferromagnetic coupling strength get enhanced and hence we observe a large magnetoresistance. The mixed valence and disordered arrangement of Fe and Co leads to complex magnetic behaviour forming the spin glass state. From neutron diffraction and magnetization measurements it was confirmed that Fe and Co in SFCO have mixed valence states. While $\mathrm{Co}^{3+}$ is in an intermediate state the other valence states are in low spin states. Preferential stability of the IS state over HS due to strong ligand metal hybridization has also been reported already [48, 49]. Hence we infer that the positive contri- bution to MR, though small, is due to the coexistence of the FM and AFM interactions. As seen in SFCO the magnetoresistive behaviour due to the enhancement of the FM regions due to the application of a magnetic field in a cluster glass system were also reported in the Co-rich phases of Co doped $\mathrm{Sr}_{2} \mathrm{FeMoO}_{6} 3$. It is instructive to compare the negative magnetoresistive value of $63 \%$ in SFCO with the case of $\mathrm{Sr}_{2} \mathrm{FeMoO}_{6}$ which shows $42 \% \mathrm{MR}$ and $\mathrm{Sr}_{2} \mathrm{CoMoO}_{6}$ with zero MR 50.

\section{Conclusions}

To summarize, the neutron diffraction study and subsequent analysis of the structural parameters confirm the tetragonal $I 4 / \mathrm{m}$ structure and reveal Jahn-Teller distortions present in SFCO double perovskite. Based on the analysis of Mössbauer and XPS spectra we substantiate the presence of mixed valency of transition metal cations which leads to an inhomogeneous magnetic state in SFCO. These studies also confirm the intermediate spin state of $\mathrm{Co}^{3+}$ in this material. Large magnetoresistance of $63 \%$ is observed at low temperatures in SFCO. Using KhoslaFischer model, it is confirmed that the MR has negative and positive components which originates from the localized magnetic moments and the spin glass state respectively.

\section{Acknowledgements}

The authors are grateful to B. R. K. Nanda for his comments and critical review in improving the manuscript. 
The magnetoresistance measurements were performed at ments. PR wishes to thank Rajeev Rawat, Ajay Gupta, IGCAR Kalpakkam. Specific heat measurements were per- $\quad$ R. Nirmala, K. Balamurugan and E. Senthil Kumar for formed at UGC-DAE Consortium for Scientific Research, stimulating discussions.

Indore, India. The authors thank A. Bharathi and A. T.

Satya, IGCAR Kalpakkam for their help in MR measure- 


\section{References}

1. D. Serrate, J.M. De Teresa, M.R. Ibarra, J. Phys.: Condens. Matter 19, 023201 (2007)

2. D.D. Sarma, E.V. Sampathkumaran, S. Ray, R. Nagarajan, S. Majumdar, A. Kumar, G. Nalini, T.N. Guru Row, Solid State Commun. 114(9), 465 (2000)

3. H. Chang, M. García-Hernández, M. Retuerto, J.A. Alonso, Phys. Rev. B 73(10), 104417 (2006)

4. A.S. Ogale, S.B. Ogale, R. Ramesh, T. Venkatesan, Appl. Phys. Lett. 75, 537 (1999)

5. A. Poddar, R. Bhowmik, I. Muthuselvam, N. Das, J. Appl. Phys. 106(7), 073908 (2009)

6. S. Baidya, T. Saha-Dasgupta, Phys. Rev. B 84(3), 035131 (2011)

7. K. Kugel and D. I. Khomskii Sov. Phys. Usp. 25231 (1982)

8. A. Maignan, C. Martin, N. Nguyen, B. Raveau, Solid State Sci. 3(1-2), 57 (2001)

9. P. Bezdicka, L. Fournés, A. Wattiaux, J.C. Grenier, M. Pouchard, Solid State Commun. 91(7), 501 (1994)

10. V. V. Bannikov, I. R. Shein, V. L. Kozhevnikov, A. L. Ivanovskii, J. Struc. Chem. 49(5), 781 (2008)

11. T. Wei, Q. Zhang, Y.H. Huang, J.B. Goodenough, J. Mater. Chem. 22, 225 (2011)

12. Pradheesh R., Harikrishnan S. Nair, C.M.N. Kumar, Jagat Lamsal, R. Nirmala, P.N. Santhosh, W.B. Yelon, S.K. Malik, K. Sethupathi, V. Sankaranarayanan, J. Appl. Phys. 111053905 (2012)

13. I.G. Deac, S.V. Diaz, B.G. Kim, S.W. Cheong, P. Schiffer, Phys. Rev. B 65(17), 174426 (2002)

14. H.M. Rietveld, J. Appl. Cryst. 2, 65 (1969)

15. J. Rodriguez-Carvajal, Physica B 192, 55 (1993)
16. J. Hjóllum and M. B. Madsen Comput. Phys.(physics.comp-ph)arxiv:0912.0449v1 (2006)

17. M.W. Lufaso, P.M. Woodward, J. Godberger, J. Solid State Chem. 177, 1651 (2004)

18. S. Satpathy, Z. Popović, F. Vukajlović, Phys. Rev. Lett. 76(6), 960 (1996)

19. M. Cheah, P.J. Saines, B.J. Kennedy, J. Solid State Chem. 179(6), $1775(2006)$

20. M.W. Lufaso, P.M. Woodward, Acta Cryst. B 57(6), 725 $(2001)$

21. Z. Németh, Z. Klencsár, A. Vértes, K. Nomura, J. Nucl. Rad. Sci. 9, R1 (2008)

22. D. Dickson, F. Berry, Mössbauer Spectroscopy (Cambridge University Press, 1986)

23. U. Shimony, J.M. Knudsen, Phys. Rev. 144(1), 361 (1966)

24. T. Motohashi, B. Raveau, M. Hervieu, A. Maignan, V. Pralong, N. Nguyen, V. Caignaert, J. Phys.: Condens. Matter 18, 2157 (2006)

25. Z. Homonnay, K. Nomura, G. Juhász, M. Gál, K. Sólymos, S. Hamakawa, T. Hayakawa, A. Vértes, Chem. Mater. 14(3), $1127(2002)$

26. L.O. Martin, J.P. Chapman, L. Lezama, J.J.S. Garitaonandia, J.S. Marcos, J. Rodríguez-Fernández, M.I. Arriortua, T. Rojo, J. Mater. Chem. 16, 66 (2006)

27. B. Liu, Y. Zhang, L. Tang, Inter. J. Hyd. Ener. 34(1), 435 $(2009)$

28. M. Oku, J. Solid State Chem. 23(1-2), 177 (1978)

29. T. Chuang, C. Brundle, D. Rice, Surf. Sci. 59(2), 413 (1976)

30. K. Tabata, I. Matsumoto, S. Kohiki, J. Mater. Sci. 22(5), 1882 (1987) 
31. F. Munakata, H. Takahashi, Y. Akimune, Y. Shichi, M. Tanimura, Y. Inoue, R. Itti, Y. Koyama, Phys. Rev. B 56(3), $979(1997)$

32. S. Harikrishnan, C.M. Naveen Kumar, H.L. Bhat, S. Elizabeth, U.K. Rößler, K. Dörr, S. Rößler, S. Wirth, J. Phys.: Condens. Matter 20, 275234 (2008)

33. J.J. Hamilton, E.L. Keatley, H.L. Ju, A.K. Raychaudhuri, V.N. Smolyaninova, R.L. Greene, Phys. Rev. B 54, 14926 (1996)

34. J.A. Mydosh, Spin Glasses: An experimental Introduction (London: Taylor and Francis, 1993)

35. D. Martin, Phys. Rev. B 20(1), 368 (1979)

36. A. Georges, O. Parcollet, S. Sachdev, Phys. Rev. Lett. $\mathbf{8 5}(4), 840(2000)$

37. A. Banerjee, R. Rawat, K. Mukherjee, P. Chaddah, Phys. Rev. B 79(21), 212403 (2009)

38. M. Tovar, J.G. Sereni, P. Pedrazzini, A. Caneiro, F. Prado, A. Butera, M.T. Causa, J. Magn. Magn. Mater. 272, 857 (2004)

39. T. Okuda, K.I. Kobayashi, Y. Tomioka, Y. Tokura, Phys. Rev. B 68(14), 144407 (2003)

40. E.S.R. Gopal, Specific heats at low temperatures (NewYork:Plenum Press, 1966)
41. C.R. Wiebe, J.E. Greedan, P.P. Kyriakou, G.M. Luke, J.S. Gardner, A. Fukaya, I.M. Gat-Malureanu, P.L. Russo, A.T. Savici, Y.J. Uemura, Phys. Rev. B 68(13), 134410 (2003)

42. M.A. Gusmao, L. Ghivelder, R.S. Freitas, R.A. Ribeiro, O.F. De Lima, F. Damay, L.F. Cohen, Solid State Commun. 127, 683 (2003)

43. Y. Toyozawa, J. Phys. Soc. Jpn. 17, 986 (1962)

44. R.P. Khosla, J.R. Fischer, Phys. Rev. B 2, 4084 (1970)

45. X.G. Luo, X.H. Chen, G.Y. Wang, C.H. Wang, X. Li, G. Wu, Y.M. Xiong, Eur. Phys. J. B 49, 37 (2006)

46. C. Ballhausen Introduction to ligand field theory $\mathbf{9 6 2}$ (1962) (McGraw-Hill New York)

47. T. Mizokawa, L.H. Tjeng, P.G. Steeneken, N.B. Brookes, I. Tsukada, T. Yamamoto, K. Uchinokura, Phys. Rev. B 64, $115104(2001)$

48. T. Saitoh, T. Mizokawa, A. Fujimori, M. Abbate, Y. Takeda, M. Takano, Phys. Rev. B 56, 1290 (1997)

49. D. Louca, J. L. Sarrao, J. D. Thompson, H. Röder and G. H. Kwei Phys. Rev. B 6010378 (1999)

50. M.C. Viola, M.J. Martínez-Lope, J.A. Alonso, P. Velasco, J.L. Martínez, J.C. Pedregosa, R.E. Carbonio, M.T. Fernández-Daz, Chem. Mater. 14(2), 812 (2002) 